\title{
Cognitive Impairment in Moderate Degree Diffuse Axonal Head Injuries: Analysis of 84 Cases Using MMSE
}

\author{
Momin Abdul Jaleel ${ }^{1}$ Shighakolli Ramesh ${ }^{1} \quad$ Subodh Raju² ${ }^{2}$ Renuka Sharma ${ }^{1}$ Shailendra Anjankar ${ }^{1}$ \\ R. Harikishore Reddy ${ }^{1}$
}

1Department of Neurosurgery, Kamineni Hospitals, Hyderabad, Telangana, India

2Department of Neurosurgery, Apollo Hospitals, Hyderabad, Telangana, India

Address for correspondence Momin Abdul Jaleel, MBBS, MCh, Department of Neurosurgery, Kamineni Hospitals, Hyderabad, Telangana, India (e-mail: drjaleelmomin@gmail.com).

Indian J Neurotrauma:2020;17:6-10

\begin{abstract}
Background Cognitive impairment is commonly seen in traumatic brain injury survivors. Posttraumatic cognitive sequelae may be more devastating than focal motor and focal sensory deficits, and are usually left unattended.

Aim and Objective Aim of this study was to assess cognitive impairment in patients who had sustained moderate degree diffuse axonal injuries and having good outcome (Glasgow Outcome Score of 5).

Methods and Materials Prospective observational study was done from 2011 to 2015 on the patients who had sustained moderated degree diffuse brain injuries. Eighty-four cases fulfilling the inclusion criteria were studied. Patients were assessed with Mini-Mental Status Examination at discharge, end of 1 month, and at 3 months. Result Seventy-six were males and 8 were females. Age ranged from 16 to 49 years. Note that $4.76 \%$ (4) patients had hypotension at presentation, $32.14 \%$ (27) patients had associated injuries, and $19.04 \%$ (16) patients had hyponatremia at presentation. Diabetes mellitus was seen in $34.52 \%$ (29) patients, while hypertension was seen in $14.28 \%$ (12). At 3 months' follow-up, 19.06\% (16) patients had cognitive impairment. The present study revealed that hypotension and presence of associated injuries

Keywords

- head injury

- diffuse axonal injury

- cognitive impairment

- Mini-Mental Status

Examination at presentation raises the odds of having cognitive impairment by 8 and 5 times, respectively.

Conclusion Routine assessment of cognitive impairment in head injury survivors is essential as it may help in identifying cognitive deficits. Early intervention of neurorehabilitation to such patients results in better neurocognitive outcome. Hypotension and associated injuries are associated with poor cognitive outcome.
\end{abstract}

\section{Introduction}

Sixty-nine million (95\% confidence interval $64-74$ million) individuals are estimated to suffer traumatic brain injury (TBI) from various causes each year, with the Southeast Asian and Western Pacific regions experiencing the greatest overall burden of the disease. ${ }^{1}$ Cognitive impairment is common in TBI survivors. It may lead to reduced efficiency, decreased effectiveness in the performance of routine activities of daily living, or failure to adapt to novel or problematic situation. ${ }^{2}$ Posttraumatic cognitive sequelae may be more devastating than focal motor and sensory deficits and are usually left unattended. Common cognitive sequelae are impairment in domains of attention, executive functions, working memory, information-processing speed, language functions, and visuospatial processing. ${ }^{3}$ 


\section{Aim and Objective}

Our aim of this study was to assess cognitive impairment in patients who had sustained moderate degree diffuse brain injury having good outcome (Glasgow Outcome Score of 5) at 3 months using Mini-Mental Status Examination (MMSE).

\section{Methods and Materials}

This is a prospective observational study done for time period from 2011 to 2015 on patients who had sustained moderate degree diffuse brain injuries. Out of 219 patients in the age group of 18 to 50 years who were admitted at our center with moderate head injury (Glasgow Coma Score [GCS] of 9-12) and computed tomography (CT) imaging showing no focal lesion like subdural hemorrhage, epidural hemorrhage, and contusions, only 84 patients who were qualified till matriculation were included in our study, to avoid bias due to educational status. Patients with mild and severe head injury, patients with cognitive impairment before injury, Alzheimer's disease, Parkinson's disease, dementia, H/O previous head injury/stroke/meningitis/encephalitis, and any other disease involving the central nervous system were excluded from the study.

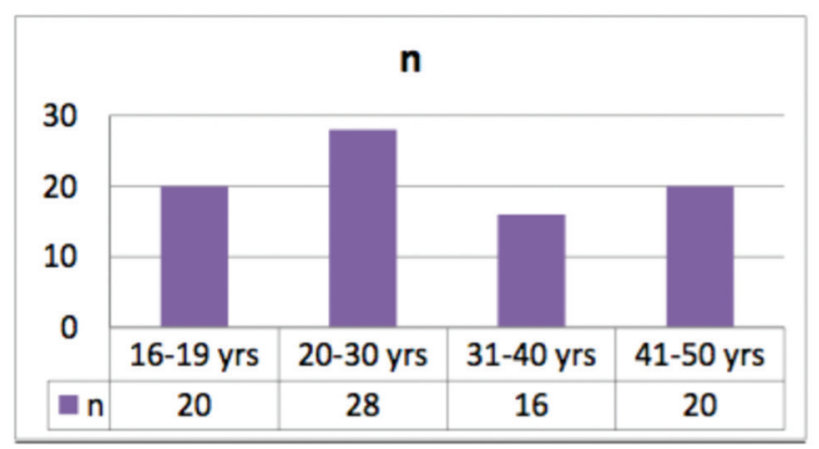

Fig. 1 Age distribution.
Age, sex, education status, mode of injury, seizures and vomiting prior to presentation, GCS at the time of presentation, baseline CT scan brain findings, hemodynamic status, hyponatremia, associated injuries (chest injury, long bone injury, abdomen solid organ injury), and comorbidities (diabetes mellitus and hypertension) were recorded at time of presentation in emergency and analyzed (-Fig. 1). Magnetic resonance imaging (MRI) of brain was done in all patients to grade the diffuse axonal injury (DAI) as described by Adams et al. ${ }^{4}$ Cognitive impairment was assessed using MMSE as it is simple, easy to administer, less time consuming, and covers all domains which are commonly affected. Score of less than 24 out of 30 was considered as cognitive impairment, and graded as mild (20-24), moderate (19-16), and severe $(<15)$ cognitive impairment as described by Folstein et al. ${ }^{5}$ Patients were assessed with MMSE at discharge, end of 1 month, and at 3 months.

Statistical Analysis: Data was tabulated and analyzed using Microsoft Excel software. Basic statistical tools like percentage and standard deviations were used. Univariate and multivariate regression analysis was done. $p$-Value of $<0.001$ was considered significant.

\section{Results}

Sixty-eight patients with DAI had better cognition, whereas other 16 had cognitive impairment. Among 84 patients, 76 were males and 8 were females. Age ranged from 16 to 49 years as shown in - Fig. 1. Seizures are seen in 33.33\% (24), vomiting in $35.71 \%$ (30), and ear, nose, and throat bleed in $30.95 \%$ (26). The most common mode of injury in our study was due to road traffic accident as seen in 90. Forty-seven percent (76) of patients and 8.52\% (8) patients had injuries due to fall. Note that $4.76 \%$ (4) patients had hypotension at presentation, 32.14\% (27) patients had associated injuries, and $19.04 \%$ (16) patients had hyponatremia at presentation. Diabetes mellitus was seen in $34.52 \%$ (29) patients, while hypertension was seen in $14.28 \%$ (12). Both diabetes mellitus and hypertension were seen in $15.47 \%$ (13) patients.

Table 1 Distributions of variables among cognitively impaired and normal

\begin{tabular}{|c|c|c|c|}
\hline & & Cognitively normal & Cognitively impaired \\
\hline & & $n=68$ & $n=16$ \\
\hline Age & Mean (SD) & $28.82( \pm 10.4)$ & $35.28( \pm 11.02)$ \\
\hline Gender (M/F) & & $64 / 4$ & $12 / 4$ \\
\hline GCS at admission (mean) & & 9.57 & 9.75 \\
\hline \multicolumn{4}{|l|}{ Comorbidities } \\
\hline DM & & 12 & 0 \\
\hline HTN & & 8 & 4 \\
\hline \multicolumn{4}{|l|}{ Mode of injury } \\
\hline Fall & & 4 & 4 \\
\hline RTA & & 64 & 12 \\
\hline Length of stay (d) & & $9.55( \pm 6.20)$ & $19.71( \pm 10.53)$ \\
\hline
\end{tabular}

Abbreviations: DM, diabetes mellitus; F, female; GCS, Glasgow Coma Score; HTN, hypertension; M, male; RTA, road traffic accident; SD, standard deviation. 
Distributions of variables among cognitively impaired and normal are shown in - Table $\mathbf{1}$.

Cognitive impairment was measured by using MMSE at discharge, 1 month, and 3 months of follow-up. At discharge, $61.90 \%$ (52) patients had cognitive impairment. Cognitive impairment was dominant in areas of orientation and memory. At 1-month follow-up, 33.33\% (28) patients had cognitive impairment. Most of the patients at 1 month had shown cognitive improvement in domain of orientation. At 3 months' follow-up, 19.06\% (16) patients out of 84 had cognitive impairment. Note that $80.94 \%$ (68) patients do not have cognitive impairment. All domains were affected at the end of 3 months in cognitively impaired patients. The cognitive impairment was significant and is represented in -Table $\mathbf{2}$.

Univariate and multivariate regression analysis of the data disclosed that age, presence of associated injuries, and hypotension at presentation was identified as the factors which can affect the cognitive outcome significantly as shown in - Table 3.

Patients having hypotension at presentation raises the odds of having cognitive impairment at the end of 3 months by 8 times, whereas presence of associated injuries at presentation raises the odds of having cognitive impairment at end of 3 months by 5 times.

\section{Discussion}

Cognitive impairment due to TBI is a substantial source of morbidity for affected individuals, their family members, and society. Disturbances of attention, memory, and executive functioning are the most common neurocognitive consequences of TBI. ${ }^{6}$ In the present study, all domains of cognition were significantly affected and difference was statically significant. Our result is consistent with Scheid et al study who demonstrated similar impairment in all domains in patients who sustained DAI. ${ }^{7}$

Many recent studies on MRI of brain with TBIs showed that microstructural changes occur within hours after the initial insult and affect the integrity of the white matter, therefore leading to the varied manifestation of neuropsychological impairments. ${ }^{8}$ And MRI lesion pattern compatible with isolated DAI is associated with persistent cognitive impairment. ${ }^{7}$ On the contrary, we found that there is gradual improvement in cognition in $19.06 \%$ of patients. Our findings were similar to those of Skandsen et $\mathrm{al}^{9}$ and Finnanger et $\mathrm{a}^{10}$ who found out that half of the patients with moderate TBI and even one-third of those with severe TBI had a normal cognitive assessment 3 months postinjury and there was improved processing speed and visual memory at 12 months. Greater cognitive reserve may be the reason for better improvement which must be showing some degree of resilience to the impact of injury. ${ }^{11}$

Moderate degree DAI patients have significant deficits of episodic memory including immediate and delayed verbal memory recall, verbal recognition, immediate and delayed visual memory recall, naming, verbal fluency, and information processing speed. The comprehensive neuropsychological assessments in all cases of TBI are important. ${ }^{12}$ There are many batteries to assess cognitive impairment of the patients with TBI. We used MMSE because of its ease of use and it covers all domains of cognition. MMSE was compared against a variety of gold standards and its reliability and construct validity were judged to be satisfactory. MMSE fulfilled its original goal of providing a brief screening test that quantitatively assesses the severity of cognitive impairment and documents cognitive changes occurring over time. ${ }^{13}$ Many authors have used it as the screening tool for assessment of cognitive impairment following brain injury..$^{14,15}$

In our study, age was statistically significant in affecting the cognitive outcome in patients which was consistent with a recent article from Brazil stating that age and level of education may influence cognition, with younger ages and higher education level playing protective roles. ${ }^{16}$

Reynolds et al and Vanderploeg et al showed age and gender were nonsignificant whereas social and economic predisposing issues had great effect on cognitive outcome after head injury. ${ }^{17,18}$ Out of eight females in the present study, four (50\%) had cognitive impairment at end of 3 months. Our result is inconsistent with most of other studies which shows that females tend to have better prognosis than males.

Table 3 Univariate and multivariate regression analysis

\begin{tabular}{|l|l|l|}
\hline Variable & OR & $95 \% \mathrm{Cl}$ \\
\hline Age & 1.0506 & $0.9849-1.1208$ \\
\hline Associated injuries & 5.4624 & $1.1696-25.5099$ \\
\hline Hypotension & 8.6934 & $0.6659-113.5004$ \\
\hline Pulse rate & 1.0394 & $0.9936-1.0873$ \\
\hline CT-grading & 0.5684 & $0.1639-1.9712$ \\
\hline
\end{tabular}

Abbreviations: $\mathrm{Cl}$, confidence interval; $\mathrm{CT}$, computed tomography; $\mathrm{OR}$, odds ratio.

Table 2 Cognitive outcome, individual domain involvement assessed by MMSE at 3 months after discharge

\begin{tabular}{|c|c|c|c|}
\hline & Cognitively normal & Cognitively impaired & $p$-Value \\
\hline & $n=68$ & $n=16$ & \\
\hline MMSE at 3 mo & $26.82( \pm 2.52)$ & $21.25( \pm 2.52)$ & $<0.001$ \\
\hline Orientation & $10( \pm 0.47)$ & $9.25( \pm 0.53)$ & $<0.001$ \\
\hline Memory & $4.65( \pm 0.79)$ & $3.25( \pm 0.47)$ & $<0.001$ \\
\hline Attention and calculation & $4.24( \pm 0.79)$ & $3.25( \pm 0.79)$ & $<0.001$ \\
\hline Language and praxia & $7.94( \pm 1.17)$ & $5.50( \pm 1.17)$ & $<0.001$ \\
\hline
\end{tabular}

Abbreviation: MMSE, Mini-Mental Status Examination. 
On assessment of factors at presentation affecting the cognitive outcome in moderate degree diffuse brain injuries, we found that age, hypotension, and associated injuries to have significantly affected the outcome. In contrary to our study, Willemse-van son et al in 2009 have shown that age, Barthel index score, and preinjury community integration scores were the major determinants of cognitive impairment at 36 months after head injury whereas cause of injury, presence of hypoxia, presence of hypotension, and hypothermia did not show any effect on outcome. ${ }^{19}$ Severe TBI and no education are usually associated with worse cognitive performance and higher disability. ${ }^{15}$ Presence of structural brain lesion adversely influence the final MMSE scores. They can be concluded to be poor prognostic factors in terms of cognitive function in TBI patients. Development of hydrocephalus on later date can aggravate cognitive impairment with unpredictable time of onset. ${ }^{14}$ The present study revealed that hypotension and presence of associated injuries at presentation raises the odds of having cognitive impairment by eight and five times, respectively. These findings are in accordance with recent study showing that a single, acute hypotensive event lasting 30 minutes did not aggravate the shortand long-term structural and motor deficits but delayed the speed of recovery of cognitive function..$^{20}$ Concomitant injuries may also increase both peripheral and central inflammatory responses and hence deficits associated with TBI may be exacerbated. ${ }^{21}$ Also, comorbidities like diabetes and hypertension leads to brain pathology in aging brain, and can result in hippocampal atrophy which may affect cognition in later life. ${ }^{22} \mathrm{TBI}$ patients with such comorbidities are more prone to cognitive impairment than there healthy counterparts.

Limitation of our study is that we have not studied patients with mild and severe DAI who are also prone for cognitive impairment. Effect of literacy on MMSE score, influence of alcohol, and hypoxia were not studied, which were excluded from the study to avoid bias.

\section{Conclusion}

Routine assessment of cognitive impairment is essential as it may help in early neurorehabilitation to such patients resulting in better neurocognitive outcome. Hypotension, associated injuries, and presence of comorbidities such as diabetes and hypertension are associated with poor cognition. So, prevention of hypotension, prompt treatment of associated injuries, and treatment of comorbidities are essential.

\section{Financial Disclosure}

None.

\section{Note}

Presentation in 64th Annual Conference of Neurological Society of India conducted in Hyderabad, 2015. Work bagged K.K. Bisaria Award for Best Paper award in Allied Neurosciences category.

\section{Acknowledgments}

Corresponding author and all coauthors are involved in research and article writing.

\section{Conflicts of Interest}

None.

\section{References}

1 Dewan MC, Rattani A, Gupta S, et al. Estimating the global incidence of traumatic brain injury. J Neurosurg 2018;1:1-18

2 Mesulam M-M, Principles of Behavioural and Cognitive Neurology. 2nd ed. Philadelphia: FA Davis; 2000

3 Chaudhury S, Pande V, Saini R, et al. Neuropsychiatric sequelae of head injury. Indian J Neurotrauma 2005;2:13-21

4 Adams JH, Doyle D, Ford I, Gennarelli TA, Graham DI, McLellan DR. Diffuse axonal injury in head injury: definition, diagnosis and grading. Histopathology 1989;15(1):49-59

5 Folstein MF, Folstein SE, McHugh PR. "Mini-mental state". A practical method for grading the cognitive state of patients for the clinician. J Psychiatr Res 1975;12(3):189-198

6 Arciniegas DB, Held K, Wagner P. Cognitive impairment following traumatic brain injury. Curr Treat Options Neurol 2002;4(1):43-57

7 Scheid R, Walther K, Guthke T. Preul C, von Cramon DY. Cognitive sequelae of diffuse axonal injury. Arch Neurol 2006;63(3):418-424

8 Veeramuthu V, Narayanan V, Kuo TL, et al. Diffusion tensor imaging parameters in mild traumatic brain injury and its correlation with early neuropsychological impairment: a longitudinal study. J Neurotrauma 2015;32(19):1497-1509

9 Skandsen T, Finnanger TG, Andersson S, Lydersen S, Brunner JF, Vik A. Cognitive impairment 3 months after moderate and severe traumatic brain injury: a prospective follow-up study. Arch Phys Med Rehabil 2010;91(12):1904-1913

10 Finnanger TG, Skandsen T, Andersson S, Lydersen S, Vik A, Indredavik M. Differentiated patterns of cognitive impairment 12 months after severe and moderate traumatic brain injury. Brain Inj 2013;27(13-14):1606-1616

11 Leary JB, Kim GY, Bradley CL, et al. The association of cognitive reserve in chronic-phase functional and neuropsychological outcomes following traumatic brain injury. J Head Trauma Rehabil 2018;33(1):E28-E35

12 Miotto EC, Cinalli FZ, Serrao VT, Benute GG, Lucia MC, Scaff M. Cognitive deficits in patients with mild to moderate traumatic brain injury. Arq Neuropsiquiatr 2010;68(6):862-868

13 Tombaugh TN, McIntyre NJ. The mini-mental state examination: a comprehensive review. J Am Geriatr Soc 1992;40(9):922-935

14 Lee CN, Koh YC, Moon CT, Park DS, Song SW. Serial minimental status examination to evaluate cognitive outcome in patients with traumatic brain injury. Korean J Neurotrauma 2015;11(1):6-10

15 Sharbafshaaer M. Impacts of cognitive impairment for different levels and causes of traumatic brain injury, and education status in TBI patients. Dement Neuropsychol 2018;12(4):415-420

16 de Freitas Cardoso MG, Faleiro RM, de Paula JJ, et al. Cognitive impairment following acute mild traumatic brain injury. Front Neurol 2019;10:198

17 Reynolds S, Paniak C, Toller-Lobe G. Nagy J. A longitudinal study of compensation-seeking and return to work in a treated mild traumatic brain injury sample. J Head Trauma Rehabil 2003;18(2):139-147 
10 Cognitive Impairment in Moderate Degree Diffuse Axonal Head Injuries Jaleel et al.

18 Vanderploeg RD, Curtiss G, Duchnick JJ, Luis CA. Demographic, medical, and psychiatric factors in work and marital status after mild head injury. J Head Trauma Rehabil 2003;18(2):148-163

19 Willemse-van Son AH, Ribbers GM, Hop WC, Stam HJ. Community integration following moderate to severe traumatic brain injury: a longitudinal investigation. J Rehabil Med 2009;41(7):521-527

20 Schütz C, Stover JF, Thompson HJ, et al. Acute, transient hemorrhagic hypotension does not aggravate structural damage or neurologic motor deficits but delays the long-term cognitive recovery following mild to moderate traumatic brain injury. Crit Care Med 2006;34(2):492-501

21 McDonald SJ, Sun M, Agoston DV, Shultz SR. The effect of concomitant peripheral injury on traumatic brain injury pathobiology and outcome. J Neuroinflammation 2016; 13(1):90

22 Roberts RO, Knopman DS, Przybelski SA, et al. Association of type 2 diabetes with brain atrophy and cognitive impairment. Neurology 2014;82(13):1132-1141 